\title{
Review
}

\section{UV-mediated Thiol-ene Polyol Functionalization for Synthesis of Biobased Waterborne Polyurethanes}

\author{
Jevgenij Lazko" ${ }^{1 *}$, Loïc Poussard ${ }^{1}$, Jérôme Mariage', Fouad Laoutid ${ }^{1}$, Jean Marie Raquez ${ }^{1,2}$, Philippe \\ Dubois $^{1,2}$ \\ ${ }^{1}$ Materia Nova Research Center, Laboratory of Polymeric and Composite Materials (SMPC), Mons 7000, Belgium. \\ ${ }^{2}$ Center of Innovation and Research in Materials \& Polymers (CIRMAP), University of Mons (UMons), Mons 7000, Belgium.
}

Correspondence to: Jevgenij Lazko, Materia Nova Research Center, Laboratory of Polymeric and Composite Materials (SMPC), Avenue Copernic 3, Mons 7000, Belgium. E-mail: jevgenij.lazko@materianova.be

Cite this article: Lazko H, Poussard L, Mariage J, Laoutid F, Raquez JM, Dubois P. UV-mediated Thiol-ene Polyol Functionalization for Synthesis of Biobased Waterborne Polyurethanes. Adv Mat/ Sci Tech2020;2:1:1-11.

Published: Jun 2020

\section{Abstract}

Developing waterborne polyurethane coatings from biobased polyols represents an interesting alternative, allowing at the same time to increase the use of sustainable renewable raw materials and to reduce volatile organic compounds emissions. In this work, biobased Veopur polyol was first functionalized with mercaptopropionic acid using solvent-free UV-mediated thiol-ene reaction performed in bulk. Grafted carboxylic moieties were then neutralized by triethylamine in order to obtain the required amphiphilic behavior. In the final step, functionalized water dispersible polyol was polymerized with water soluble polyisocyanate to form waterborne polyurethane (WPU). The influence of key-process parameters on grafting efficiency was investigated by iodometric titration, Fourier-transform infrared spectroscopy and proton nuclear magnetic resonance. Particle size measurements and stress-strain tests were carried out to characterize WPU water dispersions and corresponding materials, respectively.

Keywords

Waterborne polyurethane; biobased polyol; thiol-ene grafting; soft segment; functionalization; UV; dispersion

\section{INTRODUCTION}

The evolution of the regulatory requirements relevant to health, safety and environment is currently driving research into finding new alternatives for polyurethane-based coatings. Developing waterborne polyurethanes (WPU) was undoubtedly a major technological advance that significantly reduced volatile organic compounds emissions $\mathrm{s}^{[1,2]}$. Further sustainability improvements can be achieved replacing petro-based chemistry by renewable bio-based alternatives, using for instance polyols issued from abundant and low cost agro-industrial 
oils, and particularly of unsaturated ones ${ }^{[3-11]}$ (Desroches et al., 2012). Such biobased and water dispersable polyurethanes (bio-WPU) for coating applications were already synthetized, inter alia, from castorbean (Ricinus communis $)^{[12-14]}$, rapeseed (Brassica napus $)^{[15,16]}$, jatropha (Jatropha curcas) ${ }^{[17]}$ soybean $(\text { Glycine max })^{[18,19]}$ and linseed (Linum usitatissimum) ${ }^{[20]}$ functionalized oils.

Due to the intrinsic hydrophobicity of all previously cited plant oil-based polyols, the predominant strategy to obtain WPU consisted in hard segment functionalization by the incorporation of ionic hydrophilic segments directly into polymer backbone during the synthesis. Dimethylolpropionic acid (DMPA) neutralized with triethylamine (TEA) seems to be the most commonly used pair of charges improving biobased PU amphiphilic properties $^{[21]}$. Hard segment functionalization strategy with DMPA/TEA has been also the subject of our recent works relevant to biobased WPU for coating applications and the influence of process parameters on their physicochemical, mechanical and thermal properties ${ }^{[22]}$.

An alternative strategy, involving soft segment functionalization, consists to graft charges directly on the biobased polyols. This functionalization is expected to bring new amphiphilic properties to the polyols, enhancing their hydrophilic character and eventually their dispersability in water. Such modified polyols may finally react with diisocyanates to form WPUs. In this respect, solvent-free UV-mediated thiol-ene grafting of mercapto-carboxylic acids on polyol double bonds might represent a sustainable environmentally friendly approach fully considering intrinsic chemical assets of unsaturated vegetal oils.

UV-mediated thiol-ene chemistry has been the subject of increased interest in polymer synthesis, crosslinking and functionalization ${ }^{[23-30]}$. Crosslinked hydrogels for biomedical applications were formed via photopolymerization of functionalized PEG and bis-cysteine peptides ${ }^{[31]}$. Crosslinked networks could be also obtained upon UV exposure of polyfunctional thiols and waterborne polyurethane pre-polymers containing pendant unsaturations ${ }^{[32]}$.

The potential of UV-initiated solvent-free thiol-ene reactions, carried out without deoxygenation, at room temperature and without the use of any metal catalyst, was highlighted for the synthesis of dendrimers and well-defined functional macromolecules ${ }^{[33]}$. Photoinduced thiol-ene couplings were successfully carried out for preparation of thermoset polymers using multifunctional alkyl ester mercaptopropionates and biobased unsaturated fatty acids $^{[34]}$, macrolactones ${ }^{[35]}$ and limonene ${ }^{[36]}$. Photochemically initiated thiol-ene click couplings were compared to thermally induced reactions on a series of alkene-functional polymers such as polystyrene (PS), polycaprolactone (PCL), poly(methyl methacrylate) (PMMA) and polyethylene glycol (PEG), UV treatments systematically leading to higher yields and shorted reaction times ${ }^{[37]}$. In this study, the grafting of mercaptoacetic acid was precisely selected for its ability to add carboxylic groups in place of carbon-carbon double bounds and, consequently, to bring significant change in the solubility of functionalized polymers.

UV-mediated thiol-ene coupling strategy was also applied for the synthesis of fully-biobased waterborne polyurethanes $^{[13]}$. Fatty dicarboxylic acid (precursor which would be later transformed into diisocyanate by Curtius rearrangement) as well as hydrophilic chain extender were successfully synthetized by coupling of 3-mercaptopropionic acid with undecylenic acid and castor oil, respectively. In both cases, UV-induced thiolene couplings were carried out in solution (dichloroethane and ethanol), under stirring for 4.5 and $6 \mathrm{~h}$, resulting in yields as high as $92 \%$. Finally, after the completion of the polymerization reaction, dispersability in water of such synthesized PU was ensured by neutralization of carboxylic acid moieties by addition of triethylamine. Such a strategy to obtain the both reactants by thiol-ene reaction is of strong interest but although presents the drawback of the use of organic solvent.

Due to the low viscosity of fatty acid dimers at ambient temperature, the soft segment functionalization strategy investigated in the present study consists to graft mercaptopropionic acid (MPA) on the biobased polyol Veopur from esterified soybean oil fatty acids, using solvent-free UV-mediated thiol-ene reaction performed in bulk. Grafted carboxylic moieties were then neutralized by TEA and thus water dispersable functionalized polyol was finally polymerized with commercial water soluble polyisocyanate to form waterborne polyurethanes (WPU). The emphasis was placed on the influence of key-process parameters on grafting efficiency, modifications 
being followed by iodometric titration, fourier-transform infrared spectroscopy (FTIR) and proton nuclear magnetic resonance $\left({ }^{1} \mathrm{H}\right.$ NMR). The study also concerned the influence of grafting rate on water dispersability of functionalized polyols and properties of corresponding WPUs, characterized respectively by particle size measurements in the dispersion state and by stress-strain measurements in the solid state after water evaporation.

\section{MATERIALS AND METHODS}

\subsection{Materials}

Biobased polyol Veopur 721075 from esterified soybean oil fatty acids was supplied by Vandeputte Oleochemicals (Belgium) and used without any further purification. According to the provided analyses, hydroxyl value $\mathrm{I}_{\mathrm{OH}}(70 \mathrm{mg} \mathrm{KOH} / \mathrm{g})$ and Wijs titration iodine value $\left(102 \mathrm{~g} \mathrm{I}_{2} / 100 \mathrm{~g}\right)$ revealed the following characteristics: a molecular weight around $1600 \mathrm{~g} / \mathrm{mol}$ and 5.2 double bonds in mean per molecule. Irgacure 2022 photoinitiator, composed of phosphine oxide phenyl bis (2,4,6-trimethyl benzoyl) $20 \mathrm{wt} \%$ and 2-hydroxy2-methyl-1- phenyl-1-propanone $80 \mathrm{wt} \%$, was kindly provided by Ciba Specialty Chemicals (Germany). Mercaptopropionic acid (MPA, 99\% purity) and triethylamine (TEA, 99\% purity) were purchased from SigmaAldrich. Bayhydur 401-70, a self-emulsifiable polyisocyanate for $2 \mathrm{~K}$ WPUs coatings based on polyethylene glycol functionalized isophorone diisocyanate (IPDI) (NCO $2.34 \mathrm{mmol} / \mathrm{g}$ ) was supplied by Bayer (Germany). Methylethyl ketone (MEK), methanol $(\mathrm{MeOH})$ and isopropanol were purchased from Merck and deuterated chloroform $\left(\mathrm{CDCl}_{3}, 99.8 \% \mathrm{~d}\right)$ from Euriso-Top.

\subsection{UV Grafting Procedure}

Photochemical thiol-ene grafting was carried out in a DYMAX UV Lamp chamber (Torrington, USA), under $50-80 \mathrm{~mW} / \mathrm{cm}^{2}$ irradiation at $365 \mathrm{~nm}$, during $90-200 \mathrm{~min}$ in order to graft carboxylic moieties on fatty acid dimers [Figure 1]. Components were mixed manually before introduction in the UV chamber. The reaction was done in bulk, without any additional solvent, in glass-covered rectangular $160 \mathrm{~mm} \times 150 \mathrm{~mm} \times 10 \mathrm{~mm}$ Teflon moulds. Different MPA/Veopur ratios were investigated from 0.5 to $2(\mathrm{~mol} / \mathrm{mol})$ thiol $/$ ene ratio. Another parameter was the weight ratio of photoinitiator Irgacure/Veopur varying from $1 / 10$ to $1 / 3 \mathrm{~g} / \mathrm{g}$ (10 to $33 \mathrm{wt} \%$ ). In order to remove unreacted thiol and the residues of photoinitiator after UV treatment, modified polyol was purified by 5 consecutive liquid-liquid extractions with 2 equivalent volumes of methanol. Finally, the product was dried under vacuum at $60^{\circ} \mathrm{C}$ to a constant weight.

\subsection{Iodometric Titration}

Decrease in the amount of free thiols remaining in the reaction medium was directly followed during the UV-treatment by iodometric titration ${ }^{[38]} .500 \mathrm{mg}$ samples were dissolved in $15 \mathrm{~g} \mathrm{MEK} /$ Isopropanol $1 / 2 \mathrm{~g} /$ $\mathrm{g}$ and titrated with $0.025 \mathrm{~N}$ iodine solution. The quantity of the free thiol was calculated using the formula $n_{\text {thiol }}=N_{I_{2}} \times V_{I_{2}}$, with $N_{I_{2}}$ and $V_{I_{2}}$ representing the molarity and the volume of iodine solution, respectively. Grafting rate was then defined as SH consumed per CC double bond equivalent.

\subsection{FTIR Spectroscopy}

FTIR spectra were recorded in absorbance (3800-600 $\left.\mathrm{cm}^{-1}\right)$ at a nominal resolution of $1 \mathrm{~cm}^{-1}$ using Bruker TENSOR 27 spectrometer (Ettlingen, Germany).

\subsection{NMR Spectroscopy}

${ }^{1} \mathrm{H}$ NMR spectroscopy was performed using Bruker Avance 500 Spectrometer (Billerica, USA) at a frequency of $500 \mathrm{MHz}$ to confirm each step of polyol functionalization on samples dissolved in $\mathrm{CDCl}_{3}(20 \mathrm{mg} / 600 \mu \mathrm{L})$.

\subsection{Particle Size Distribution}

WPU dispersions were characterized by dynamic light scattering measurements using Malvern Instruments Zetasizer Nano-S (Malvern, UK). Samples were systematically diluted by 100 to reach $0.2 \mathrm{wt} \%$ solid content and analyzed at $20^{\circ} \mathrm{C}$. All measurements were performed in triplicate.

\subsection{Mechanical Properties}

Tensile properties [Young modulus $(\mathrm{E})$, the stress at break $\left(\sigma_{\mathrm{b}}\right)$ and the strain at break $\left(\varepsilon_{\mathrm{b}}\right)$ ] were evaluated 


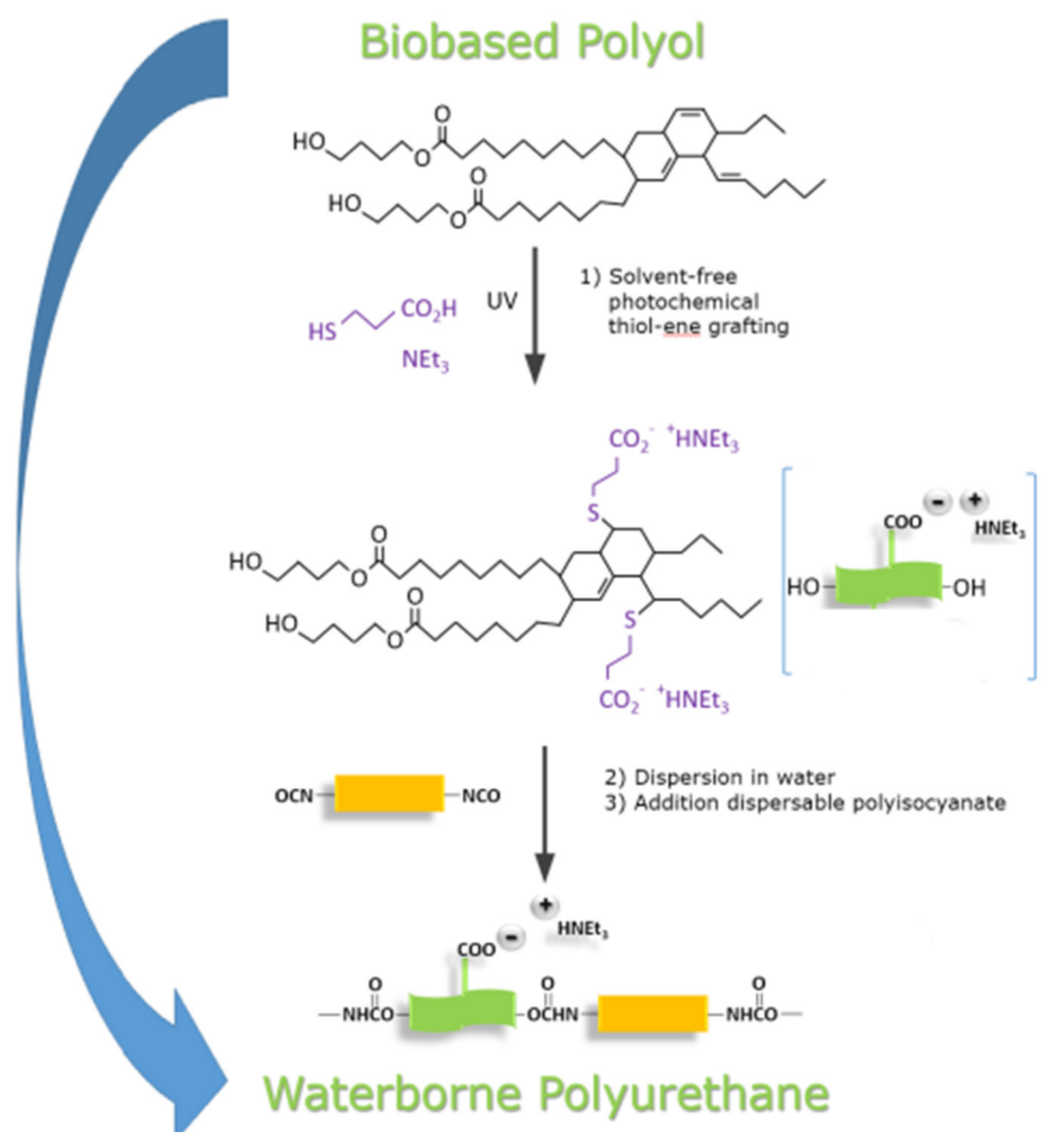

Figure 1. Reaction scheme for synthesis of waterborne polyurethane through UV-mediated functionalization of biobased Veopur polyol.

according to the ASTM D638 standard on dumbbell-shaped samples cut from the WPU sheets at $10 \mathrm{~mm} \cdot \mathrm{min}^{-1}$ using a Benelux Scientific Hounsfield H10KT apparatus (Nazareth, Belgium) equipped with 100 N load cell.

\section{RESULTS AND DISCUSSION}

\subsection{Main UV Treatment Parameters}

Concentration of the photoinitiator, initial thiol/ene ratio and UV intensity are three key-parameters influencing grafting kinetics that have been studied in this work. First, the influence of UV intensity was evidenced comparing $50 \mathrm{~mW} / \mathrm{cm}^{2}$ and $80 \mathrm{~mW} / \mathrm{cm}^{2}$ treatments [Table 1]. Grafting kinetics and final grafting rates were increased from $43 \%$ to $51 \%$ with the increase of the UV intensity from 50 to $8 \mathrm{~mW} / \mathrm{cm}^{2}$, for the set of trials at low Irgacure concentration (10\%). The overall increase in grafting efficiency was also noticed at high Irgacure 


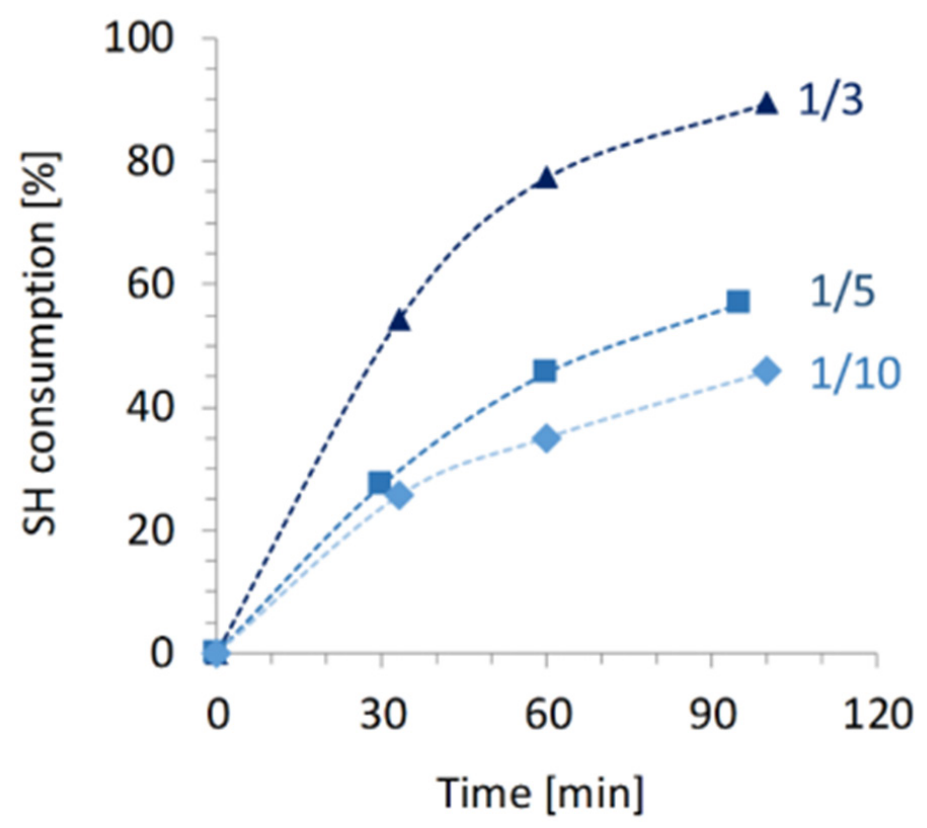

Figure 2. Influence of the initial photoinitiator concentration on MPA consumption kinetics. $(1 / 10,1 / 5$ and $1 / 3$ Irgacure 2022/Veopur $\mathrm{g} / \mathrm{g}$, $4 \mathrm{~g}$ samples, $1 / 1$ thiol/ene $\mathrm{mol} / \mathrm{mol}, 50 \mathrm{~mW} / \mathrm{cm}^{2}$ ).

concentration (33\%), reaching $95 \%$. However, in the context of this study, UV intensity of $50 \mathrm{~mW} / \mathrm{cm}^{2}$ has been found to be more suitable for further investigation and up-scaling trials, primarily due to UV device specificities and also to avoid heat generation phenomena leading to undesirable secondary decompositions reactions.

The influence of the photoinitiator concentration on UV-mediated grafting kinetics was clearly shown using $1 / 10,1 / 5$ and $1 / 3 \mathrm{~g} / \mathrm{g}$ initial Igracure 2022/Veopur ratios for low-amount $(4 \mathrm{~g}$ ) Veopur samples prepared in small circular $40 \mathrm{~mm}$ moulds [Figure 2]. When treated at $50 \mathrm{~mW} / \mathrm{cm}^{2}$ with $1 / 1$ thiol/ene, higher conversion rates around $90 \%$ could be reached in 100 min using 33\% Irgacure media. For the scale-up of the polyol production (up to $60 \mathrm{~g}$, vide infra, section 3.2), the initial photoinitiator content was consequently chosen at $33 \mathrm{wt} \%$ of the introduced polyol.

Initial thiol/ene ratio is also an essential parameter influencing grafting kinetics and grafting rates [Table 2]. As reported in literature, most researches on thiol-ene reactions were performed with a high excess of thiol, with two or more thiol functions per carbon-carbon double bond ${ }^{[39]}$. In this work, we used a derivative method ${ }^{[40]}$ and investigated a reduced range of thiol/ene ratio, to limit the thiol excess to be removed afterwards. Different sets of modified polyols, covering a wide range of grafting rates from $47 \%$ to $98 \%$, were obtained at $50 \mathrm{~W} / \mathrm{cm}^{2}$, by varying thiol/ene initial ratio from 0.5 to 2 . Clear relationship can be observed between initial thiol/ene ratio, absolute thiol consumption and grafting rate (thiol consumption relative to the theoretical amount of carboncarbon double bonds). Lower 0.5 thiol/ene ratios trigger higher thiol consumptions, MPA preferentially reacting with the most accessible carbon-carbon double bonds, i.e., conversion rate being close to the theoretical value of $50 \%$. For stoichiometric thiol/ene ratio, an average conversion rate of $67 \%$ was obtained. Finally, high grafting rates superior to $95 \%$ could be reached in presence of a large excess of thiol.

Accordingly, the optimized conditions to reach high conversion rates with $4 \mathrm{~g}$ Veopur samples were found at $50 \mathrm{~mW} / \mathrm{cm}^{2}$ irradiation using 33\% Irgacure/Veopur media and 1/1 thiol/ene $\mathrm{mol} / \mathrm{mol}$ ratio. In the next section, similar operational conditions were used for upscaling the process, in order to obtain functionalized polyols in quantities allowing further polymerization and characterization tests from being performed.

\subsection{MPA Grafting Upscale}

MPA grafting UV treatments were performed in larger $160 \mathrm{~mm} \times 150 \mathrm{~mm} \times 10 \mathrm{~mm}$ rectangular moulds 
Table 1. Impact of UV intensity and photoinitiator concentration on grafting rates, determined by iodometric titration. 4 g Veopur samples, 1/1 thiol/ene $\mathrm{mol} / \mathrm{mol}$ ratio, $90 \mathrm{~min}$

\begin{tabular}{lll}
\hline $\mathbf{U V}$ intensity $\left(\mathbf{m W} / \mathbf{c m}^{\mathbf{2}}\right)$ & Photoinitiator/Polyol $\mathbf{( g / g )}$ & Grafting rate $\mathbf{( \% )}$ \\
\hline 50 & $1 / 10$ & 43 \\
80 & $1 / 10$ & 51 \\
50 & $1 / 3$ & 86 \\
80 & $1 / 3$ & 95 \\
\hline
\end{tabular}

Table 2. Influence of the initial thiol/ene ratio on thiol consumption and grafting rate, determined by iodometric titration. $30 \mathrm{~g}$ Veopur samples, 1/3 Irgacure 2022/Veopur g/g, $50 \mathrm{~mW} / \mathrm{cm}^{2}, 180 \mathrm{~min}$

\begin{tabular}{lll}
\hline Thiol/ene (mol/mol) & Absolute thiol consumption (\%) & Grafting rate (\%) \\
\hline 0.5 & 94 & 47 \\
0.67 & 79 & 53 \\
1.0 & 67 & 67 \\
1.2 & 71 & 86 \\
2.0 & 49 & 98 \\
\hline
\end{tabular}

Table 3. Upscaling of the UV treatment. Grafting rates determined by iodometric titration and obtained with 30 and $60 \mathrm{~g}$ Veopur samples $\left(1 / 1\right.$ thiol/ene $\mathrm{mol} / \mathrm{mol}$ ratio, $\left.50 \mathrm{~mW} / \mathrm{cm}^{2}\right)$

\begin{tabular}{llll}
\hline Polyol (g) & Photoinitiator/Polyol (g/g) & Time (min) & Grafting rate (\%) \\
\hline 30 & $1 / 10$ & 180 & 64 \\
30 & $1 / 5$ & 180 & 72 \\
30 & $1 / 3$ & 180 & 81 \\
60 & $1 / 10$ & 200 & 46 \\
60 & $1 / 5$ & 200 & 58 \\
60 & $1 / 3$ & 200 & 74 \\
\hline
\end{tabular}

containing up to $60 \mathrm{~g}$ of Veopur [Table 3]. Major upscaling bottlenecks, such as heterogeneous UV intensity in the UV chamber, heat accumulation and mass loss, have been overcome by improving the experimental device, homogenizing the reaction medium and extending the treatment time up to 200 min. For instance, due to the experimental device geometry inducing a heterogeneous UV exposure, grafting rates measured at the end of the treatment could locally vary by a factor of two, only depending on the sample position in the center or the periphery of the mould. Hence, it was possible to increase up to $7 \%$ the overall grafting rate by several homogenizations of the reaction medium during the treatment, spaced out by $45 \mathrm{~min}$.

As previously observed, the grafting rates considerably increased with the Irgacure content. Depending on photoinitiator concentration, different ranges of grafting rates between $64 \%$ - $81 \%$ and between $46 \%$ - $74 \%$ were obtained with 30 and 60 g production scale, respectively. More interestingly, the lower grafting rates for upscale modified polyol, whatever the photoinitiator content, would be assumed to the higher thickness of the mixture limiting the UV beam penetration.

The impact of UV grafting treatments on structural modifications was first investigated by FTIR [Figure 3]. The grafting rate could be recorded by following the increase of the MPA characteristic absorption bands at 1440 $\mathrm{cm}^{-1}, 1340 \mathrm{~cm}^{-1}$ and in the $1250-1150 \mathrm{~cm}^{-1}$ region, corresponding to $-\mathrm{CH}_{2}-\mathrm{S}-\mathrm{R}$ and $-\mathrm{COO}-$ bonds ${ }^{[40-44]}$.

Quantitative structural studies of the modified polyols were carried out by ${ }^{1} \mathrm{H}$ NMR. Specific resonance signals were identified for each reagent, i.e., Veopur, MPA and Irgacure 2022, and were normalized with respect to Veopur methylene proton signal at $0.85 \mathrm{ppm}$ (which always remained unchanged, before or after grafting). Purification efficiency ( 5 washing steps with $\mathrm{MeOH}$ ) was verified for all samples: integration of Irgacure 2022 specific signals in 8.0-7.9 ppm domain, corresponding to aromatic protons, proved systematically close to the detection limits and the baseline noise. For MPA, emphasis was placed onto 3.0-2.4 ppm specific domain corresponding to $-\mathrm{CH}_{2}-$ in $\alpha$ and $\beta$ positions of $\mathrm{S}$ atom of the grafted MPA [Figure 4]. Due to the overlap of 

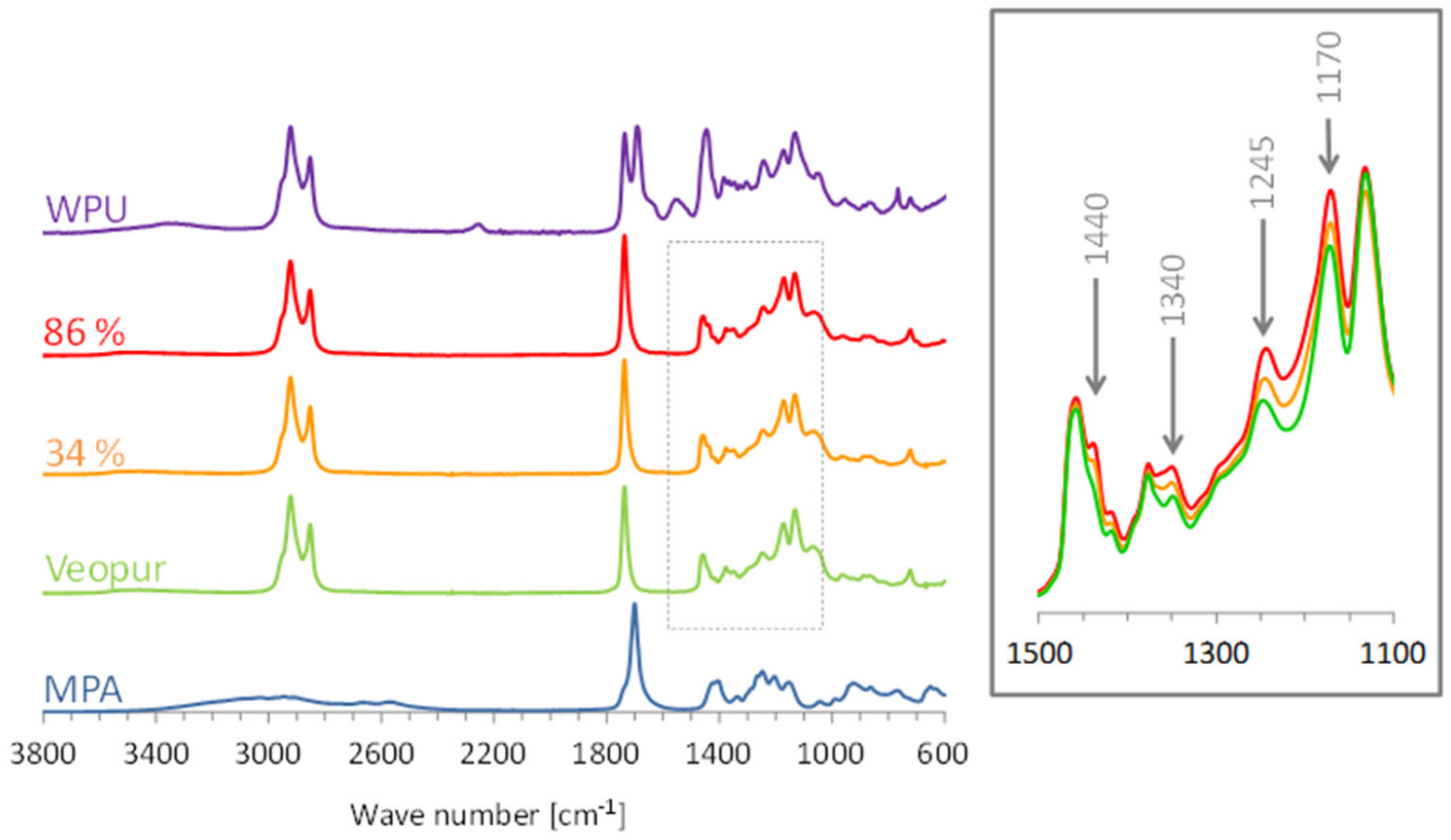

Figure 3. FTIR spectra of MPA, Veopur, functionalized Veopur after UV thiol-ene grafting and final waterborne polyurethane.
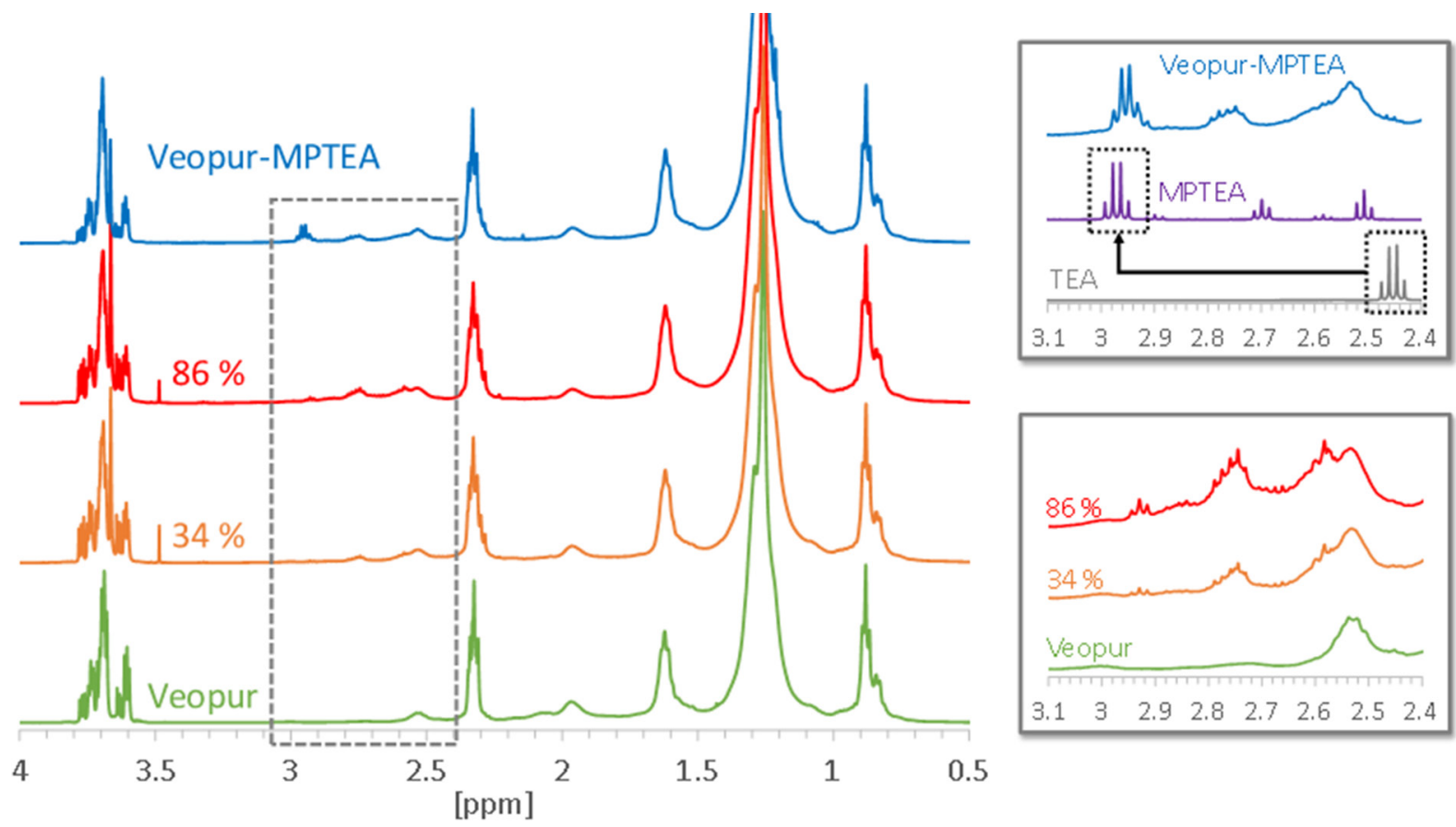

Figure 4. ${ }^{1} \mathrm{H}$ NMR spectra of the reference Veopur, MPA-grafted Veopur and MPA-grafted Veopur neutralized with TEA (coded as Veopur-MPTEA).

Veopur aliphatic signal at 2.6-2.5 ppm, the final integration of the grafted MPA was obtained after subtraction of the respective integration of (MPA + Veopur) minus integration of (Veopur). ${ }^{1} \mathrm{H}$ NMR was also considered as a suitable analytical technique to confirm TEA neutralization of MPA-grafted Veopur, as the major resonance 


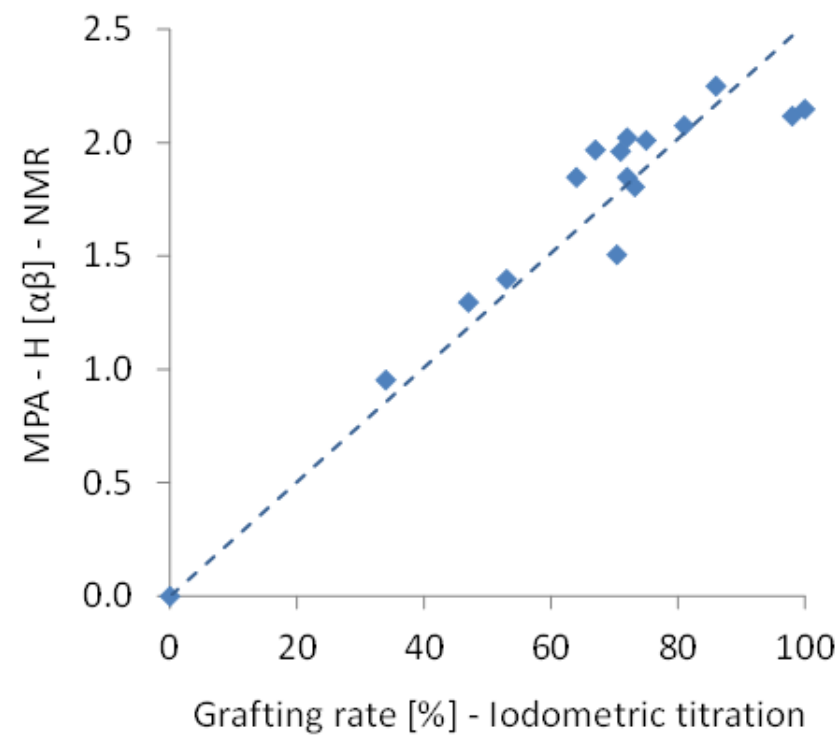

Figure 5. Relationship between 1H NMR integrations and iodometric titration results obtained for the set of MPA grafting experiments.

signals corresponding to grafted MPTEA were perfectly identifiable in the final product: $-\mathrm{S}_{-} \mathrm{CH}_{2}-\mathrm{CH}_{2}-\mathrm{COO}^{-}$ protons at $2.75 \mathrm{ppm}$ (in $\alpha$ position of $\mathrm{S}$ atom) specific to the grafted MPA and $-\mathrm{CH}_{2}$ - protons at $2.95 \mathrm{ppm}$ specific to neutralized TEA.

Finally, results obtained by iodometric titration have been compared to ${ }^{1} \mathrm{H}$ NMR data [Figure 5]. Complementarity between these two approaches, indicating respectively thiol consumption and grafting efficiency, seemed to be verified for all MPA/Veopur/Irgacure trials described in this study. A linear relation between titration results and ${ }^{1} \mathrm{H}$ NMR integration of $-\mathrm{CH}_{2}$ - of the grafted MPA in the 3.0-2.4 ppm region may be established for the whole set of grafting rates between $35 \%$ and $100 \%$.

\subsection{WPU Synthesis}

The main objective of this last part of the work was to confirm that functionalized polyols could be dispersed in water and finally polymerized. The previous modified polyols were neutralized by the stoichiometric addition of TEA relative to the grafted carboxylic acid groups. MEK was added to reduce viscosity $(50 \mathrm{wt} \%)$ and then it was possible to disperse them in water after phase-inversion and to obtain $20 \mathrm{wt} \%$ waterborne polyol dispersions [Figure 1]. Water self-emulsifiable Bayhydur polyisocyanate was then added in excess, isocyanate index $\mathrm{I}_{\mathrm{NCO}}$ varying from 110 to 130 , and the reaction medium kept under magnetic stirring for $18 \mathrm{~h}$ at $20{ }^{\circ} \mathrm{C}$. Final WPU dispersions were poured into Teflon moulds and the dry materials, i.e., WPU coatings, were finally recovered after 2 days evaporation at room temperature, followed by 1 day drying under reduced pressure in a vacuum oven at $40{ }^{\circ} \mathrm{C}$. Polymerization advancement was confirmed by FTIR by the presence of characteristic -NHCO ${ }^{-}$stretching band of urethane at $1550 \mathrm{~cm}^{-1}$ [Figure 3].

For Veopur-MPA samples, preliminary tests showed that grafting of - $\mathrm{COOH}$ functions alone was not sufficient to endow the expected amphiphilic properties to the initially highly hydrophobic polyol. On the other hand, under the form of carboxylate - $\mathrm{COO}^{-}$, stable over two months Veopur-MPTEA suspensions were obtained, with mean particle size in the range of 150-200 $\mathrm{nm}$ [Table 4]. After the polymerization step, particle mean diameter remained broadly of the same order, around $180 \pm 40 \mathrm{~nm}$. Concerning particle size distribution, at this stage of the study it was not possible to clearly conclude about an eventual impact of the grafting rate, neither for the polyol dispersions, nor for the WPU dispersions. However, the grafting rate seemed to have a significant impact on dispersion stability. The influence was evaluated for samples in the range from $35 \%$ to $98 \%$, and it was observed that a minimum grafting rate of $60 \%$ was required to obtain homogeneous and stable over time WPU dispersions without any sludge or phase separation. Moreover, WPU dispersions with lower grafting 
Table 4. Particle size of waterborne Veopur-MPTEA polyols and corresponding polyurethanes ( 0.2 wt $\%$ dispersions in water)

\begin{tabular}{lll}
\hline & \multicolumn{2}{c}{ Mean particle size (nm) } \\
\cline { 2 - 3 } Grafting rate (\%) & Veopur-MPTEA & WPU \\
\hline 46 & $202 \pm 6$ & $160 \pm 11$ \\
72 & $165 \pm 2$ & $151 \pm 3$ \\
82 & $159 \pm 2$ & $220 \pm 10$ \\
\hline
\end{tabular}

Table 5. Mechanical characterization of WPU materials. Strain-stress tests performed at $10 \mathrm{~mm} / \mathrm{min}$

\begin{tabular}{|c|c|c|c|c|}
\hline Grafting rate (\%) & $I_{\text {NCO }}$ & E (Mpa) & $\sigma_{b}(\mathrm{Mpa})$ & $\varepsilon_{\mathrm{b}}(\%)$ \\
\hline 70 & 130 & $0.292 \pm 0.031$ & $0.336 \pm 0.031$ & $141 \pm 18$ \\
\hline 72 & 110 & $0.383 \pm 0.052$ & $0.341 \pm 0.005$ & $126 \pm 23$ \\
\hline 72 & 130 & $0.447 \pm 0.060$ & $0.232 \pm 0.159$ & $137 \pm 8$ \\
\hline
\end{tabular}

rates exhibited poor coalescence during drying and led to heterogeneous materials, unsuitable for example for coating applications.

Finally, the mechanical properties of dried WPU were evaluated by stain-stress experiments. Table 5 summarizes values of Young modulus $(E)$, stress at break $\left(\sigma_{b}\right)$ and strain at break $\left(\varepsilon_{b}\right)$ obtained for several representative homogeneous and continuous WPU samples with grafting rates around $70 \%$. According to these results, all tested materials showed ductile behavior with elongation at break higher than 100\%. Therefore, our general strategy to functionalize biobased polyols and then synthetize polyurethanes in the aqueous medium, seemed to be validated since it enabled formation of ductile materials, opening perspectives for further coating developments.

\section{CONCLUSION}

Functionalization of biobased Veopur polyol by grafting mercaptopropionic acid was described in this study. Thiol-ene reaction was successfully performed in bulk using solvent-free UV-mediated process with Irgacure 2022 photoinitiator, reaching an average conversation rate of $70 \%$ for stochiometric thiol/ene ratio, and values higher than $95 \%$ with thiol excess. Neutralization of grafted carboxylic functions with triethylamine created enough charges to bring amphiphilic behavior to the initially highly hydrophobic substrate, accordingly offering functionalized polyol dispersable in water.

Polymerization of such functionalized biobased polyol with self-dispersable Bayhydur 401-70 polyisocyanate eventually led to waterborne polyurethanes. Suitable grafting rates, above $60 \%$ were however necessary to obtain fine stable waterborne polyurethane dispersions, with an average particle size around $180 \mathrm{~nm}$. Finally, homogeneous and continuous materials were obtained after drying, with particularly interesting ductile mechanical behavior, i.e., elongations at break being systematically superior to $100 \%$, setting a path toward further coating development studies.

Further polyol functionalization studies might concern improvement of grafting kinetics via development of continuous UV processes. Selection of biobased polyols, self-emulsifiable polyisocyanates and urethane catalysts might be investigated to synthetize waterborne polyurethanes with tunable mechanical properties for specific applications. For further studies concerning coating applications, the emphasis should also be placed on other specific requirements such as adhesive strength, surface properties, water swelling and thermal stability.

\section{ACKNOWLEDGEMENTS}

This research was supported by the Cornet Program in the frame of the "Cost-effective biopolymer interior coatings" Biocoat project (Convention $\left.n^{\circ} 1317997\right)$. The authors are grateful for the support of the European Union and Wallonia with the European Funds for Regional Development FEDER 2014-2020. The authors would like to thank Sabine Rossit and Cédric Deherripon (Vandeputte Oleochemicals) for supplying biobased polyol. J.-M. Raquez is "chercheur qualifié" by the F.R.S.-FNRS, Belgium. 


\section{REFERENCES}

[1] Noble KL. Waterborne polyurethanes. Progress Organic Coatings 1997,32:131-136.

[2] Dieterich D. Aqueous emulsions, dispersions and solutions of polyurethanes; synthesis and properties. Progress Organic Coatings 1981,9:281-340.

[3] Desroches M, Escouvois M, Auvergne R, Caillol S, Boutevin B. From Vegetable Oils to Polyurethanes: Synthetic Routes to Polyols and Main Industrial Products. Polymer Rev 2012,52;38-79.

[4] Lligadas G, Ronda JC, Galià M, Cádiz V. Renewable polymeric materials from vegetable oils: a perspective. Materials Today 2013,16:337-343.

[5] Noreen A, Zia KM, Zuber M, Tabasum S, Zahoor AF. Bio-based polyurethane: An efficient and environment friendly coating systems: A review. Progress Organic Coatings 2016,91:25-32.

[6] Li Y, Luo X, Hu S. Polyols and Polyurethanes from Vegetable Oils and Their Derivatives. SpringerBriefs Green Chemistry Sustainability 2015,15-43.

[7] Alam M, Akram D, Sharmin E, Zafar F, Ahmad S. Vegetable oil based eco-friendly coating materials: a review article. Arabian J Chem 2014,7:469-479.

[8] Zafar F, Ghosal A, Sharmin E, Chaturvedi R, Nishat N. A review on cleaner production of polymeric and nanocomposite coatings based on waterborne polyurethane dispersions from seed oils. Progress Organic Coatings 2019,131:259-275.

[9] Liang H, Feng Y, Lu J, Liu L, Yang Z, Luo Y, Zhang Y, Zhang C. Bio-based cationic waterborne polyurethanes dispersions prepared from different vegetable oils. Industrial Crops Products 2018,122:448-455.

[10] Ghasemlou M, Daver F, Ivanova EP, Adhikari B. Polyurethanes from seed oil-based polyols: A review of synthesis, mechanical and thermal properties. Industrial Crops Products 2019,142:111841.

[11] Sahoo S, Mohanty S, Nayak SK. Biobased polyurethane adhesive over petroleum based adhesive: use of renewable resource. J Macromolecular Sci 2018,55:36-48.

[12] Chang CW, Lu KT. Natural castor oil based 2-package waterborne polyurethane wood coatings. Progress Organic Coatings 2012,75:435-443.

[13] Fu C, Zheng Z, Yang Z, Chen Y, Shen L. A fully bio-based waterborne polyurethane dispersion from vegetable oils: from synthesis of precursors by thiol-ene reaction to study of final material. Progress Organic Coatings 2014,77:53-60.

[14] Liang H, Liu L, Lu J, Chen M, Zhang C. Castor oil-based cationic waterborne polyurethane dispersions: storage stability, thermophysical properties and antibacterial properties. Industrial Crops Products 2018,117:169-178.

[15] Philipp C, Eschig S. Waterborne polyurethane wood coatings based on rapeseed fatty acid methyl esters. Progress Organic Coatings 2012,74:705-711.

[16] Bullermann J, Friebel S, Salthammer T, Spohnholz R. Novel polyurethane dispersions based on renewable raw materials-Stability studies by variations of DMPA content and degree of neutralisation. Progress Organic Coatings 2013,76:609-615.

[17] Saalah S, Abdullah LC, Aung MM, Salleh MZ, Awangbiak DR, Basri M, Jusoh ER. Waterborne polyurethane dispersions synthesized from jatropha oil. Industrial Crops Products 2015,64:194-200.

[18] Lu Y, Larock RC. Soybean oil-based, aqueous cationic polyurethane dispersions: Synthesis and properties. Progress Organic Coatings 2010,69:31-37.

[19] Lu Y, Larock RC. Soybean-Oil-Based Waterborne Polyurethane Dispersions: Effects of Polyol Functionality and Hard Segment Content on Properties. Biomacromolecules 2008,9:3332-3340.

[20] Chang CW, Lu KT. Linseed-oil-based waterborne UV/air dual-cured wood coatings. Progress Organic Coatings 2013,76:1024-1031.

[21] Wicks ZW, Wicks DA, Rosthauser JW. Two package waterborne urethane systems. Progress Organic Coatings 2002,44:161-183.

[22] Poussard L, Lazko J, Mariage J, Raquez JM, Dubois P. Biobased waterborne polyurethanes for coating applications: How fully biobased polyols may improve the coating properties. Progress Organic Coatings 2016,97:175-183.

[23] Hoyle CE, Lee TY, Roper T. Thiol-enes: Chemistry of the past with promise for the future. J Polymer Sci Part A: Polymer Chem 2004,42:5301-5338.

[24] Lowe AB. Thiol-ene "click" reactions and recent applications in polymer and materials synthesis. Polymer Chem 2010,1:17-36.

[25] Machado TO, Sayer C, Araujo PHH. Thiol-ene polymerisation: a promising technique to obtain novel biomaterials. Eur Polymer J 2017,86:200-215.

[26] Ionescu M, Radojčić D, Wan X, Petrović ZS, Upshaw TA. Functionalized vegetable oils as precursors for polymers by thiol-ene reaction. Eur Polymer J 2015,67:439-448.

[27] Wang Z, Liang H, Yang H, Xiong L, Zhou J, Huang S, Zhao C, Zhong J, Fan X. UV-curable self-healing polyurethane coating based on thiol-ene and Diels-Alder double click reactions. Progress Organic Coatings 2019,137:105282.

[28] Aoyagi S, Shimasaki T, Teramoto N, Shibata M. Bio-based polymer networks by thiol-ene photopolymerization of allylated l-glutamic acids and 1-tyrosines. Eur Polymer J 2018,101:151-158.

[29] Xu D, Cao Z, Wang T, Zhong J, Zhao J, Gao F, Luo X, Fang Z, Cao J, Xu S, Shen L. An ambient-cured coating film obtained via a Knoevenagel and Michael addition reactions based on modified acetoacetylated castor oil prepared by a thiol-ene coupling reaction. Progress Organic Coatings 2019,135:510-516.

[30] Tajima K, Iwamoto K, Satoh Y, Sakai R, Satoh T, Dairi T. Advanced functionalization of polyhydroxyalkanoate via the UV-initiated thiol-ene click reaction. Applied Microbiol Biotechnol 2016,100:4375-4383.

[31] Aimetti AA, Machen AJ, Anseth KS. Poly(ethylene glycol) hydrogels formed by thiol-ene photopolymerization for enzyme-responsive protein delivery. Biomaterials 2009,30:6048-6054. 
[32] Otts DB, Heidenreich E, Urban MW. Novel waterborne UV-crosslinkable thiol-ene polyurethane dispersions: Synthesis and film formation. Polymer 2005,46:8162-8168.

[33] Killops KL, Campos LM, Hawker CJ. Robust, Efficient, and Orthogonal Synthesis of Dendrimers via Thiol-ene "Click" Chemistry. J Am Chem Society 2008,130:5062-5064.

[34] Claudino M, Johansson M, Jonsson M. Thiol-ene coupling of 1,2-disubstituted alkene monomers: the kinetic effect of cis/trans-isomer structures. Eur Polymer J 2010,46:2321-2332.

[35] Claudino M, Van Der Meulen I, Trey S, Jonsson M, Heise A, Johansson M. Photoinduced thiol-ene crosslinking of globalide/ $\varepsilon$-caprolactone copolymers: curing performance and resulting thermoset properties. J Polymer Sci Part A: Polymer Chem 2012,50:1624.

[36] Claudino M, Mathevet JM, Jonsson M, Johansson M. Bringing d-limonene to the scene of bio-based thermoset coatings via free-radical thiol-ene chemistry: macromonomer synthesis, UV-curing and thermo-mechanical characterization. Polymer Chem 2014,5:3245-3260.

[37] Campos LM, Killops KL, Sakai R, Paulusse JMJ, Damiron D, Drockenmuller E, Messmore BW, Hawker CJ. Development of Thermal and Photochemical Strategies for Thiol-Ene Click Polymer Functionalization. Macromolecules 2008,41:7063-7070.

[38] Boutevin G, Ameduri B, Boutevin B, Joubert JP. Synthesis and use of hydroxyl telechelic polybutadienes grafted by 2-mercaptoethanol for polyurethane resins. J Applied Polymer Sci 2000,75:1655-1666.

[39] Lowe AB. Thiol-ene "click" reactions and recent applications in polymer and materials synthesis: a first update. Polymer Chem 2014,5:4820-4870.

[40] He M, Jiang S, Xu R, Yang J, Zeng Z, Chen G. Facile functionalization of soybean oil by thiol-ene photo-click reaction for the synthesis of polyfunctional acrylate. Progress Organic Coatings 2014,77:868-871.

[41] Zhang Y, Maxted J, Barber A, Lowe C, Smith R. The durability of clear polyurethane coil coatings studied by FTIR peak fitting. Polymer Degradation Stability 2013,98:527-534.

[42] Bhargava S, Kubota M, Lewis RD, Advani SG, Prasad AK, Deitzel JM. Ultraviolet, water, and thermal aging studies of a waterborne polyurethane elastomer-based high reflectivity coating. Progress Organic Coatings 2015,79:75-82.

[43] Patel CJ, Mannari V. Air-drying bio-based polyurethane dispersion from cardanol: Synthesis and characterization of coatings. Progress Organic Coatings 2014,77:997-1006.

[44] García-pacios V, Costa V, Colera M, Martín-martínez JM. Waterborne polyurethane dispersions obtained with polycarbonate of hexanediol intended for use as coatings. Progress Organic Coatings 2011,71:136-146. 